\title{
The application of fluid mobility modelling in wireless cellular networks
}

\author{
Ronan J. Skehill* and Sean McGrath \\ Wireless Access Research Centre, University of Limerick, Ireland
}

\begin{abstract}
Mobility models, synthetic or trace, try to accurately model the movement of a single user or a group of users. Models can be used in simulators and emulators to investigate the consequences of mobility on new protocols or network management techniques. A limitation with current trace mobility models is they are based on empirical data which are limited to specific network types and environments. Limitations with synthetic models are that they are complex, computationally heavy, and lack realism. To address these issue a new approach needs to be taken. One such approach is the use of fluid mechanics and transport theory to represent user mobility. A model based on viscous free irrotational fluid mechanics with empirical data from pedestrian and vehicular studies provide a means of creating realistic group movement characteristics with smooth non random trajectories and smooth continuous velocity. The model is used in an example to provide boundary crossing rates for users in a cellular network and optimising the size of cellular location areas.
\end{abstract}

Keywords: Potential fluid flow, mobility, location area optimisation, wireless cellular networks

\section{Introduction}

In a wireless cellular [?] network mobile users are considered to have pedestrian or vehicular mobility characteristics. A mobility model is used to describe the characteristics of a single user or a set of users. From Camp et al. [?] mobility models are classified as either trace or synthetic models. Trace models are those that have been created from monitoring real life users in their environment. Trace models provide accurate network specific information on the mobility of the user. Large scale network traces are hard to come by as collecting and collating information is a repetitious and tedious task requiring substantial amount of time and effort. Most available traces are for small areas such as an indoor office environment [?]. Available traces are often proprietary as each are carried out by operators trying to get a better understanding of their systems signalling and traffic management. If a trace is available, any modification that may reflect future mobility characteristics is difficult to achieve without distorting the validity of the collected information. Synthetic models on the other hand are freely available - but often lack realism. An advantage of synthetic models is that they are flexible to incorporate possible future characteristics of mobile users without distorting the validity of the model.

Models such as the Random waypoint [?], Gaussian [?], Random Walk [?,?] in principle depend on speed and direction. Other models such as Zonoozis' model [?] takes the past movement of the user into consideration. Researchers in the telecommunication field have used these models in network simulators to evaluate new network protocols, management techniques and so forth, however the recent studies have

\footnotetext{
${ }^{*}$ Corresponding author. Tel.: +353 61 202402; Fax: +353 61 202572; E-mail: ronan.skehill@ul.ie.
} 
shown the models lack realism. There is need for a new generation of mobility models that can represent accurately user characteristics.

This paper expands on the concepts outlined in [?] and presents a synthetic model namely PotFlow. The model provides a smooth trajectory for an individual user or a group of users that incorporates empirical data from pedestrian and motor vehicular studies. The PotFlow model inherently has velocity vectors built into these trajectory streams. The velocity changes with time in a continuous manner, where in comparison with the Fluid model, the velocity changes discretely. The paper presents the model, proposes a generic cellular framework and applications of the new model.

The paper is structured as follows: Section two summarises how potential fluid mechanics can be applied to characterise mobility of pedestrians or vehicles. Section three presents the fundamentals and assumptions of the PotFlow mobility model, furthermore section three derives solutions of the Laplace stream function equation and Laplace velocity potential equation namely the FreeStream, Vortex, Doublet, Source and Sink. These solutions are building blocks in the PotFlow model for creating motorway lane and pedestrian walkway sections. The fourth section demonstrates cellular network planning applications using the proposed PotFlow model to provide boundary crossing rates for different cell environments. Finally, conclusions are drawn in the fifth section.

\section{Fluid mechanics of traffic}

There are two approaches for modelling users as a fluid; free particle flow and the continuum flow approach. Henderson [?] and Marchal [?] view pedestrian behaviour as a free particle flow rather than a continuum flow. Hughes [?] presented large crowds as a continuum, and derives a Eurlian model that governs the movement and behaviour of the crowd. The continuum model alleviates a major problem in understanding crowds, namely that a crowd needs to be treated as an identity. Similarly for vehicular traffic, several gas-kinetic such as the [?,?,?] models have been used to represent traffic flow but the vast majority of models are over complex and require several parameters to be acquired. A simplification of the gas-kinetic model is used as the foundation of macroscopic models; that is the contributions of vehicles driving with velocity $v$ having continuous attributes $w$ are aggregated. In broad terms:

$$
\int_{v} \int_{w} \text { gaskinetic } \rightarrow \text { macroscopic }
$$

These continuous attributes reflect desired velocity, desired angle-of-movement, and acceleration times. Concerning the aforementioned models, established research has focused on achieving better safety for the user, for example, in high density crowd environments it is critical to understand how people move, react and so forth to avoid any injury or loss of life. When modelling the movement of users in wireless networks, it is assumed the user (walking on the street) is not part of a crowd with a dangerously high density level (that is $>6$ people $/ \mathrm{m}^{2}$ )[?]. Therefore, assumptions are made with the type of fluid that is used in the proposed mobility model.

\subsection{Fluid mechanics and model hypotheses}

By a fluid it is meant a substance that flows, the contrary is a material that does not have this property; a solid. Although this definition is unequivocal, in real-life the distinction between a solid and fluid can often be questionable since they are many materials that exhibits characteristic behaviour of both 
solids and fluids. Most common materials can be broadly categorised according to the simple criterion as whether they flow.

In turn fluids can be classified as either a liquid or a gas. Liquids are essentially incompressible as their volumes do not change. The term hydrodynamics is often used to describe the science of incompressible fluids in motion. Gases on the other hand are compressible, and their volume can be easily changed by variation in pressure. Relating these criteria in user mobility is fairly straightforward. Users can be classified as a fluid constantly moving and are only prevented from moving by external forces. A single users volume does not change, but the density of the fluid can change.

When it comes to deducing mathematically models of fluids and equations that govern fluid motion two fundamentally different approaches are used: the Eulerian description and the Lagrangian description. The Lagrangian method divides the fluid into limitingly small 'particles', then considers the motion of each particle in response to the forces acting on it, including forces exerted by neighbouring particle. The Eulerian approach is to work directly with fields such as $\rho(x, t), v(x, t)$, and $p(x, t)$, and not try to keep track of individual fluid particles.

Given the hypothesis that fluid particles are considered to be users in a flow, the next hypothesis is regarding the description of fluid the particles reside in. The principle purpose of fluid dynamics is to predict what kind of fluid motion to expect to find for a given fluid configuration that experiences known internal and external forces. Fluids are described by three attributes: (i) Chemical Composition, (ii) Viscosity and (iii) Equation of State. Definition of these terms can be found in [?, p. 21].

\subsection{Kinematics of fluids in motion}

The mechanical state of a fluid can be either static or dynamic. If a fluid is at rest everywhere, then the liquid is considered to be static. In this case the appropriate theory is called hydrostatics. If the fluid flow is that of a dynamic one, the fluid can be steady or unsteady. If the fluid flow parameters are functions of space but not functions of time, then the flow is described as steady, or time stationary. Mathematically this is expressed by partial derivatives with respect to time of any fluid variable $f$ being identically zero.

$$
\frac{\partial f}{\partial t}=0
$$

All fluids (static, and dynamic) undergo changes in volume under changes of pressure and temperature. The capacity for changes in the density of a mass element of a fluid is known as compressibility. It is well known that gases are more easily compressed than liquids. When a fixed mass of a fluid changes in volume, its density also changes. Under normal circumstances, in fluids, the changes in density attributable to pressure are practically unobservable. Density changes due to small temperature fluctuations are negligible. In general liquids have negligible or nil changes. An incompressible fluid is one whose elements undergo no changes in volume or density.

A fluid differs from a solid in that a fluid never comes into equilibrium with a shearing force. For Newtonian viscous fluids the shear stress is proportional to the velocity gradient, and the constant of proportionality is called the coefficient of viscosity. For many applications viscosity can often be ignored, i.e., assumed to be zero, and in this case the fluid is described as inviscid.

\section{PotFlow mobility model}

Using the Eulerian description of fluid motion, an inviscid fluid with users represented by fluid particles. The fluid provides a means of creating group movement characteristics with smooth non 
random trajectories and smooth continuous velocity. The new mobility model has been called PotFlow as it is based on potential flow dynamics.

\subsection{Potential flow}

All real fluids have viscosity. However, in many cases it is reasonable to neglect the effects of viscosity. It is therefore appropriate and useful to investigate the dynamics of a fluid with no viscosity and incompressible. A fluid of this kind is called a potential flow. Potential flow does not include all the characteristics of flows that are encountered in the real world and therefore the analysis is simpler than viscous flows. Starting from this point and considering the fluid has zero viscosity, objects in the fluid are not subjected to rotational forces. This can be expressed as:

$$
\nabla \times \overrightarrow{v_{u}}=0
$$

Since the volume of the fluid remains constant it is clear that zero divergence exists:

$$
\nabla \cdot \overrightarrow{v_{u}}=0
$$

where $\vec{v}_{u}$ is a fluid velocity vector. The potential fluid flow is used for the basis of the PotFlow mobility model with the following assumptions.

\subsection{User and fluid assumptions}

The PotFlow model uses a fluid characterised by the three attributes in Section 2.1 with some assumptions made. The following assumptions outline the characteristics of the user in the flow:

- The flow is considered to be incompressible. This assumption is also applied to the multiple user case for simplicity as the fundamentals of the PotFlow are used in presenting both lane and walkway sections. This allows the linear addition of flows for both sections.

- Users are considered to be particles in a general flow, the medium is unimportant - the chemical composition is therefore neglected.

- The flow is steady and inviscid, this means the user in the flow will not be affected by any external force. Vorticity is not present in the flow.

- No external force on a single mobile user in an environment, the density of the user does not change, i.e., pressure and temperature has no effect on the Equation of State of the flow.

In analysing the PotFlow fluid flow model it is useful to visualise flow patterns. This can be done by drawing lines joining points of equal velocity - velocity contours or streamlines. When fluid is flowing past a solid boundary, e.g. the surface of a wall, fluid obviously does not flow into or out of the surface. Close to the boundary wall the streamlines must be parallel to the boundary. At all points the direction of the streamline is the direction of the fluid velocity.

In steady flow, the position of streamlines do not change. Because the fluid is moving in the same direction as the streamlines, fluid cannot cross a streamline, furthermore streamlines cannot cross each other. If they were to cross this would indicate two different velocities at the same point which is not physically possible. This point implies the particles of fluid starting on one streamline will stay on that same streamline throughout the fluid. Mathematically modelling the fluid flow, it is useful to use stream and velocity functions. The functions give the magnitude, direction and characteristics of a particle in a fluid flow, the following section illustrates the stream and velocity functions. 


\subsection{Stream and velocity potential}

Solutions to the Laplace equation of the stream function give the fundamental building elements of the PotFlow mobility model. Combinations of stream function solutions are used to create a path for a flow of users. A full derivation of these functions can be found in Appendix 1. Equation (5) is known as the Laplace equation of the stream function.

$$
\nabla^{2} \psi=0
$$

and Eq. (6)

$$
\nabla^{2} \phi=0
$$

is the Laplace equation for the velocity potential. The velocity potential is orthogonal to the stream function at all points along it. A stream function can be considered to be a line passing through every point in the fluid where the flow velocity is non-zero. The stream function gives information about the direction of fluid flow, but not about the magnitude of the flow velocity.

Five solutions of the Laplace equation of the stream function and the velocity potential; Free-stream, Sink, Source, Vortex and Doublet are presented in this paper and illustrated in the following sections.

\subsubsection{Free-stream}

The Free-stream is a real life flow by itself as it represents users travelling on a straight line with constant velocity. It can be used to replicate the fluid model which is based on Boltzman gas-kinetic theory. Let $u_{\infty}$ and $v_{\infty}$ be the velocity components $(>0)$ along the $\mathrm{x}$-axis and y-axis respectively. Solving the stream function gives:

$$
\psi_{F}=u_{\infty} y-v_{\infty} x
$$

whilst solving the velocity potential gives:

$$
\phi_{F}=u_{\infty} y+v_{\infty} x
$$

\subsubsection{Source and sink}

Coupled together are the stream function solutions that give the Source and Sink elements. A Source is similar to the concept of a garden sprinkler, it sprays water out equally in all directions at a rate of $Q$ units per unit time from the origin. If the magnitude of the sprinklers velocity vector is reversed it operates as a Sink. For either a Source or Sink, the radial velocity $v_{r}$ component is $\frac{Q}{2 \pi r}$ and its tangential velocity vector component $v_{\theta}$ is zero. Solving the Laplace equation for the stream function on the origin gives:

$$
\psi_{S}=\frac{Q}{2 \pi} \theta+C_{1}
$$

the constant $C_{1}$ can be ignored.

Solving the Laplace equation for the velocity potential on the origin gives:

$$
\phi_{S}=-\mu \ln r+C_{2}
$$

where $\mu$ is $\frac{Q}{2 \pi}$. Again, the constant $C_{2}$ can be ignored. The magnitude of $Q$ determines whether the solution is a source or sink, $+Q$ is a source, and $-Q$ is sink. 


\subsubsection{Doublet}

Doublets are source-sink pairs that are separated initially by distance $\delta$, which are brought closer by making $\delta \rightarrow 0$. In order to stop the sink from cancelling out the source and vice versa, their strengths $Q$ is increased so that $Q \delta$ is a constant $k$. This constant is called the Doublet strength. The radial velocity of a doublet is $v_{r}=-\frac{k}{2 \pi r^{2}} \cos \theta$ and the tangential velocity component is $v_{\theta}=-\frac{k}{2 \pi r^{2}} \sin \theta$. Thus the solution to the stream function on the origin gives:

$$
\psi_{D}=-\frac{k}{2 \pi} \frac{\sin \theta}{r}
$$

The solution to the velocity potential on the origin gives:

$$
\phi_{D}=-\frac{k}{2 \pi} \frac{\cos \theta}{r}
$$

In certain circumstances the flow created around a doublet is the same as a cylinder shaped object placed in a flow.

\subsubsection{Vortex}

If the streamlines and the equipotentials in a source are reversed then the source becomes a Vortex. The stream function solution presents a flow field associated with an isolated vortex of strength $\Gamma$. A vortex does not have a radial velocity component i.e. $v_{r}=0$, only the tangential component $v_{\theta}$ is present which is equal to $\frac{\Gamma}{2 \pi r}$. Therefore, solving this for the stream function gives:

$$
\psi_{V}=-\frac{\Gamma}{2 \pi} \ln (r)
$$

Solving this for the velocity potential gives at the origin gives:

$$
\phi_{V}=\frac{\Gamma}{2 \pi r} \theta
$$

Where $r$ represents the distance between the position of the vortex and the point where the stream function is evaluated. Also the sign of the vortex is important, a counter-clockwise vortex is considered "positive".

\subsection{Solutions on a point}

Figures 1, 2, 3, 4 graphically show the solutions to the Laplace equation of the stream function. The solutions described are ones located on the origin but, real environments like in a pedestrian walkway, several items may be located on or near the walkway, items such as seats, light-poles, telephone booths or simply static persons. Including more objects will force the user to change direction and speed. Table 1 outlines the solutions not on the origin, with co-ordinates of $\vec{x}=\left(x_{0}, y_{0}\right)$.

\subsection{Linearity properties}

The solutions by themselves, except the free-stream, are not valid user mobility models. When the solutions are used together they form streamlines that represent a valid user mobility model. Calculating 
Table 1

Solutions to the Velocity Potential and Stream Function not on the Origin $\vec{x}=\left(x_{0}, y_{0}\right)$

\begin{tabular}{lcc}
\hline Element & Stream Function & Velocity Potential \\
\hline Free-stream & $\psi_{f}=u_{\infty}-v_{\infty}$ & $\phi_{f}=u_{\infty}-v_{\infty}$ \\
Source & $\frac{Q}{2 \pi} \tan ^{-1} \frac{y-y_{0}}{x-x_{0}}$ & $\frac{Q}{2 \pi} \ln \left|\vec{x}-\overrightarrow{x_{0}}\right|$ \\
Sink & $\frac{Q}{2 \pi} \tan ^{-1} \frac{y-y_{0}}{x-x_{0}}$ & $\frac{Q}{2 \pi} \ln \left|\vec{x}-\overrightarrow{x_{0}}\right|$ \\
Vortex & $-\frac{\Gamma}{2 \pi} \ln \left|\vec{x}-\overrightarrow{x_{0}}\right|$ & $\frac{\Gamma}{2 \pi} \tan ^{-1} \frac{y-y_{0}}{x-x_{0}}$ \\
Doublet & $-\frac{k}{2 \pi} \frac{y-y_{0}}{\left(x-x_{0}\right)^{2}+\left(y-y_{0}\right)^{2}}$ & $\frac{k}{2 \pi} \frac{x-x_{0}}{\left(x-x_{0}\right)^{2}+\left(y-y_{0}\right)^{2}}$ \\
\hline
\end{tabular}

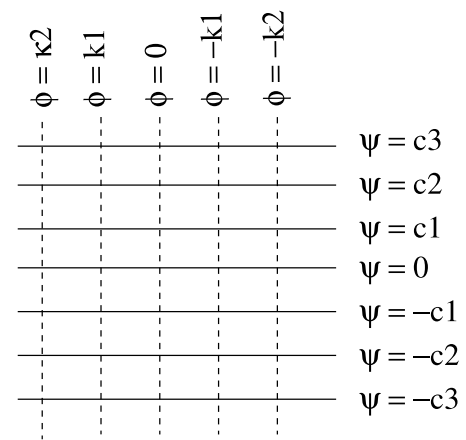

Fig. 1. Free-stream Flow Source.

a combined stream function $\psi_{c}$ is straightforward as the solutions to the Laplace equation of stream functions are linearly additive i.e.:

$$
\psi_{c}=\psi_{F}+\psi_{S}+\psi_{V}
$$

A more general form of this can be expressed as:

$$
\psi_{c}=\sum\left(\psi_{x}\right)
$$

Where $\psi_{x}$ is a fundamental stream function solution of the source, sink, vortex and doublet. This is illustrated in Fig. 5.

\subsection{Velocity field of PotFlow users}

To calculate the velocity field of PotFlow users (or fluid particle), consider a user within the PotFlow fluid at point $P$.

The velocity of point $P$ of a fluid at time $t$ is defined to be the velocity of the fluid particle at time $t$ coincides with the point $P$, this denoted by $q=q(r, t)$. In Cartesian form this is $q=q(x, y, z, t)$. This approach has been used in deriving the PotFlow solutions and the same technique is used to calculate the velocity of a PotFlow solution. Take the example of an irrotational flow formed from the superposition of a free-stream and a doublet, calculating the velocity field is straightforward. The stream function is:

$$
\begin{aligned}
\psi & =\psi_{F}+\psi_{D} \\
& =-\frac{Q}{2 \pi} \sin \theta+u_{\infty} y \\
& =-\frac{Q}{2 \pi} \sin \theta+u_{\infty} r \sin \theta
\end{aligned}
$$




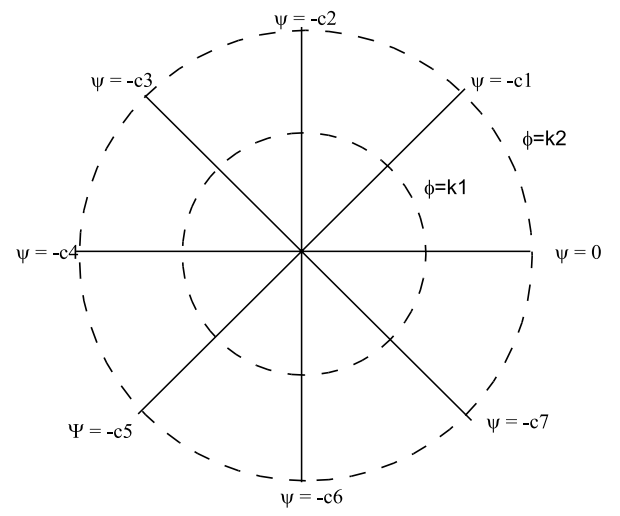

(a) sink

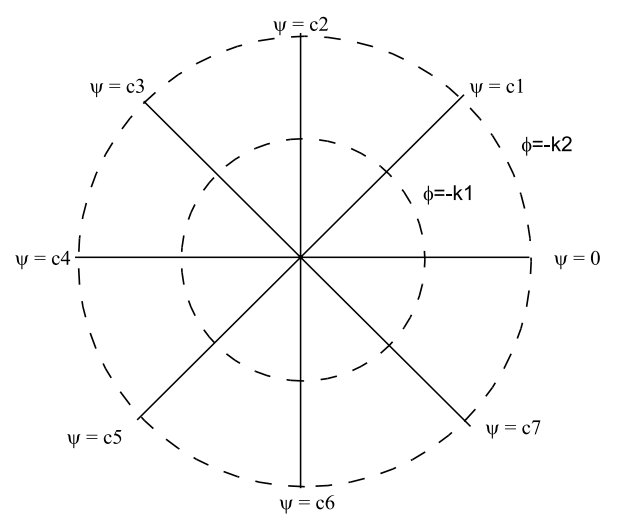

(b) source

Fig. 2. Sink and Source.

And the corresponding velocity potential is given as:

$$
\phi=\phi_{F}+\phi_{D}=-\frac{Q}{2 \pi}-u_{\infty} r \cos \theta
$$

The velocity components $\left(V_{\theta}, V_{r}\right)$ are obtained from the partial derivatives of the velocity potential:

$$
\begin{aligned}
& V_{r}=-\frac{\partial \phi}{\partial r}=-\frac{Q}{2 \pi} \frac{\cos \theta}{r^{2}}+u_{\infty} \cos \theta \\
& V_{\theta}=-\frac{1}{r} \frac{\partial \phi}{\partial \theta}=-\frac{Q}{2 \pi} \frac{\sin \theta}{r^{2}}-u_{\infty} \sin \theta
\end{aligned}
$$

The velocity field can be expressed as:

$$
\vec{V}=V_{r} \hat{e}_{r}+V_{\theta} \hat{e}_{\theta}
$$

For a free-stream and a doublet combination:

$$
\vec{V}=\left(-\frac{Q}{2 \pi} \frac{\cos \theta}{r^{2}}+u_{\infty} \cos \theta\right) \hat{e}_{r}
$$




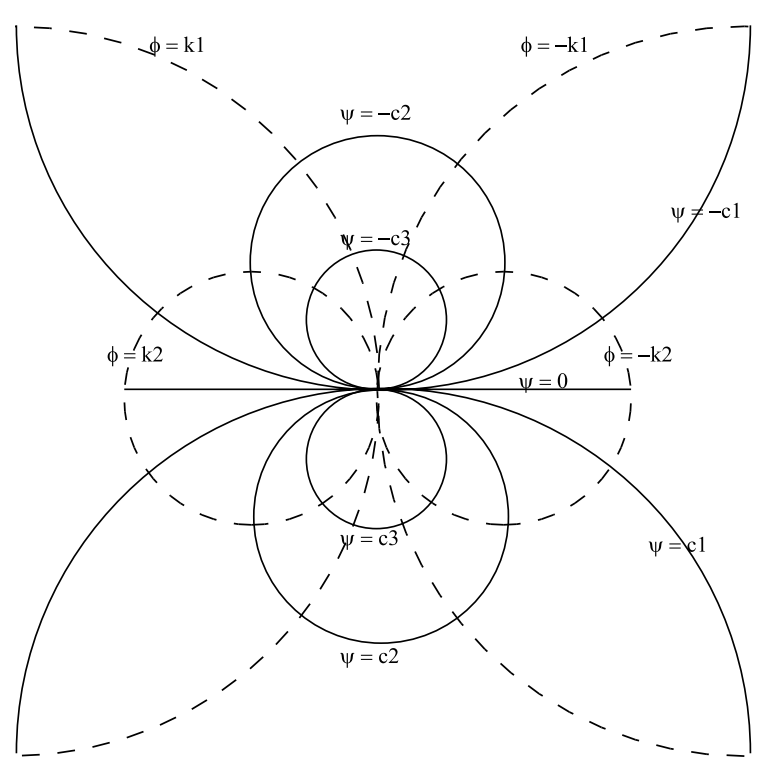

Fig. 3. Doublet.

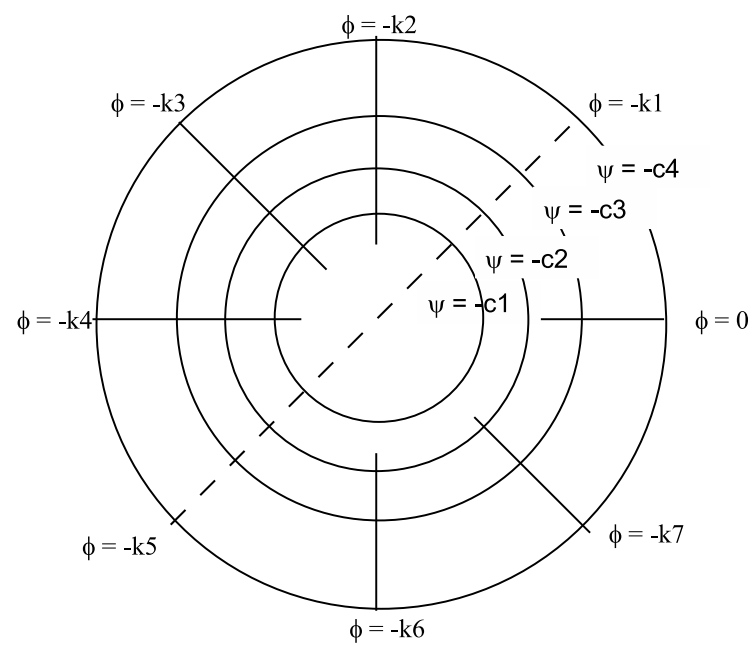

Fig. 4. Vortex.

$$
+\left(-\frac{Q}{2 \pi} \frac{\sin \theta}{r^{2}}-u_{\infty} \sin \theta\right) \hat{e}_{\theta}
$$

The magnitude of the velocity field is

$$
|\vec{V}|=\sqrt{\left(-\frac{Q}{2 \pi} \frac{\cos \theta}{r^{2}}+u_{\infty} \cos \theta\right)^{2}+\left(-\frac{Q}{2 \pi} \frac{\sin \theta}{r^{2}}-u_{\infty} \sin \theta\right)^{2}}
$$

It is clear from Eq. (22), the further away from the doublet solution, the less influence it has on the free-stream speed. This applies not only for doublets, but all fundamental solutions. This affect can 


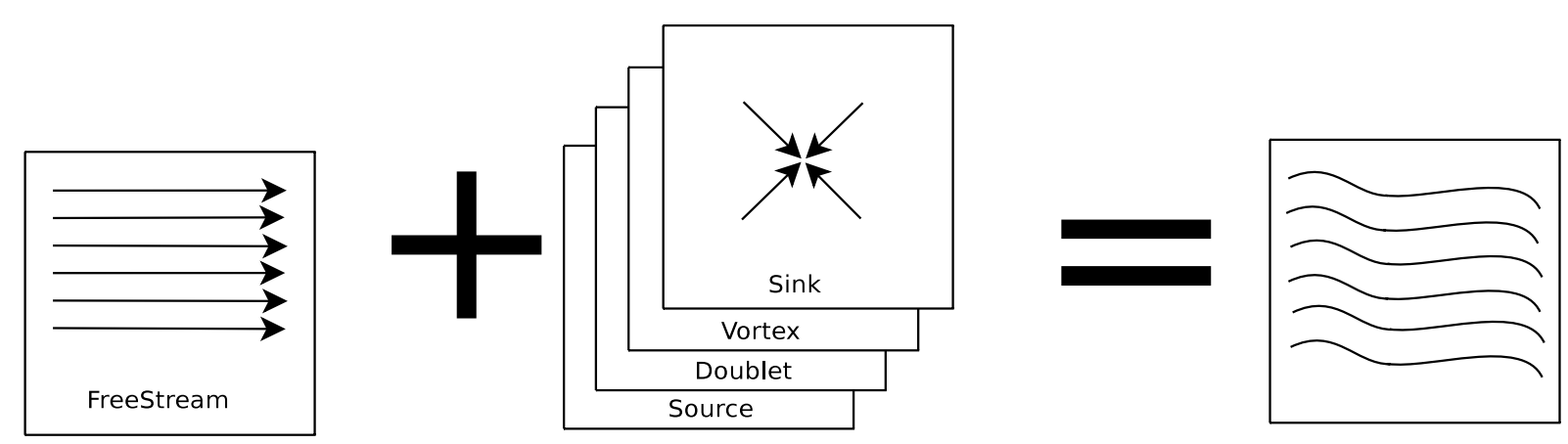

Fig. 5. Combinations of Vortex, Doublet, Sink and Source with the FreeStream give Smooth Continuous Paths with Associated Smooth Changes in Velocity.

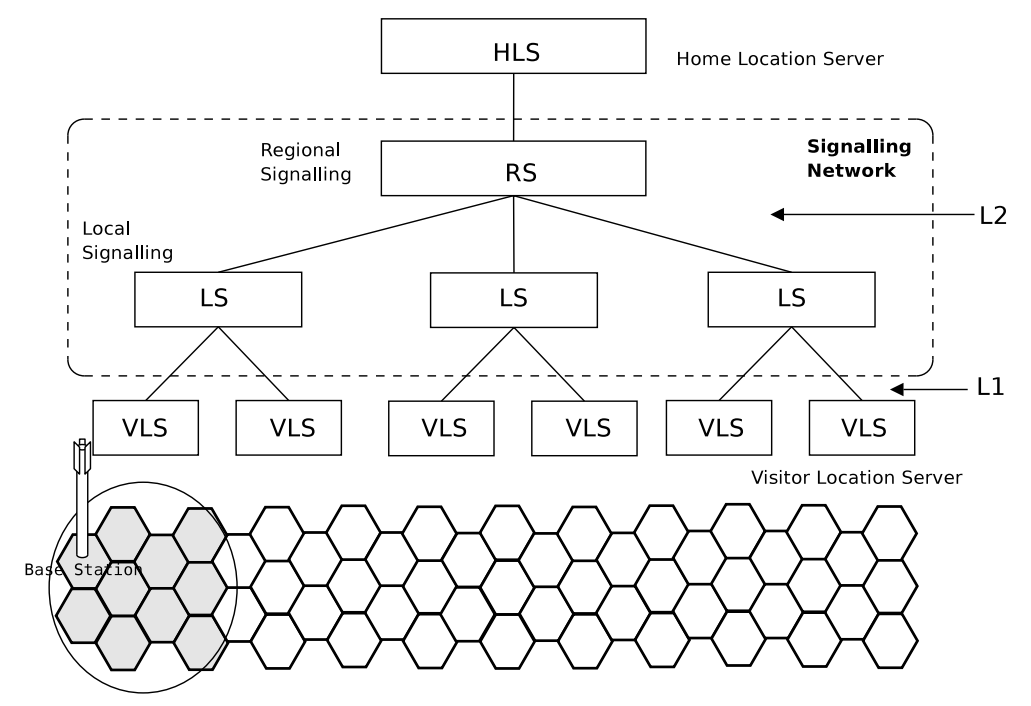

Fig. 6. A Generic Cellular Architecture.

decrease or increase free-flow speed which correlates to such effects in Transport theory. For example, consider a walkway that has a piece of street furniture (a street post), a user walking in direct line of the street furniture will try to avoid it. To avoid the obstacle the user will have to change direction and speed. In comparison to a user walking on the same walkway but away from the street post, this user will respond to the change of direction of the first user but won't have to make such a severe change in speed. With PotFlow the change in speed is smooth in comparison to the abrupt speed changes in the fluid or random walk mobility models, which gives more realism to model user behaviour. The magnitude of the velocity field can be set with empirical values obtain from [?,?].

\section{Application of the mobility model}

Mobility models are used in various calculation tasks in wireless networks, one such task is the calculation of signalling cost associated with network mobility management [?,?] techniques. As a user moves in a cellular network, it leaves and joins location areas, the user is unaware of such changes 
but using location registration the node informs the network of its current location. Location areas are clusters of cells and is assigned a unique location area identification. When a call is to be terminated at the node, the network must find the cell in the location area which the node resides in. Presented in this paper is a framework where users move according to the PotFlow mobility model and the associated signalling cost for a mobility management technique is calculated. The signalling cost is then used to calculate optimised location area sizes for macro, micro and pico cell sizes.

\subsection{Cellular network framework}

The wireless cellular framework (see Fig. 6) consists of a cellular network having a total number of cells $N_{t}$. The cell configuration is based on a regular hexagon layout [?] with no overlap of cells and with no gap between cells. The Radio Station System (RSS) is in communication with mobile nodes via the Radio Access Technology, the mobile is allowed to move in and out of cells and location areas, furthermore the mobile is able to enter idle and active states. The RSS has control over a single cell and it's located in the centre of the cell. The RSS is connected to the Core Network via some signal switching network. When a call arrives at the Core Network it checks the Home Location Server for an entry, when the HLS returns the location area (i.e. the Visitor Location Server), it begins the paging process for the called mobile node. Three environmental scenarios will be considered: pico cell environment (radius $<100 \mathrm{~m}$ ), micro cell environment (radius $100 \mathrm{~m}-1000 \mathrm{~m}$ ) and the macro cell environment (radius $1 \mathrm{~km}-5 \mathrm{~km})$.

\subsection{Optimised location area partition}

Using the numerical computing environment MATLAB the cellular framework and PotFlow mobility model is developed. The following sections describe the movement model and mobility mangement parameters used to derive the optimised location area partition.

\subsubsection{Node and movement model}

The user density $\rho$ of the cell is constant in all cases, and so too is the amount of users making a call (nodes in an active state and their exact cell location is known) $E_{c}$ when the user has to make a location update. The mobile node is paged when a call arrives for it at the core network. The velocity and trajectories of the nodes are determine by the aggregate PotFlow mobility model and [?,?].

\subsubsection{Mobility management parameters}

The selected mobility management technique in the wireless framework (illustrated by Fig. 7), is the blanket paging technique, and the registration process is the distance based [?] technique. Using the mobility model it is possible to determine the optimised size of a location area. The total cost of mobility management technique is given by Eq. (23):

$$
C_{t o t}=C_{p}+C_{u}
$$

Where the cost of Blanket Paging $C_{p}$ is:

$$
C_{p}(N)=\rho N A_{\text {cell }} \lambda T_{p} B_{p}
$$

The cost of the distance based location registration techniques $C_{u}$ is proportional to the boundary crossing rate of PotFlow nodes and is given by Eq. $(24,25)$ :

$$
C_{u}=\frac{\rho v_{x} L_{p r m}}{\pi} T_{u} B_{u}\left(1-E_{c}\right)
$$



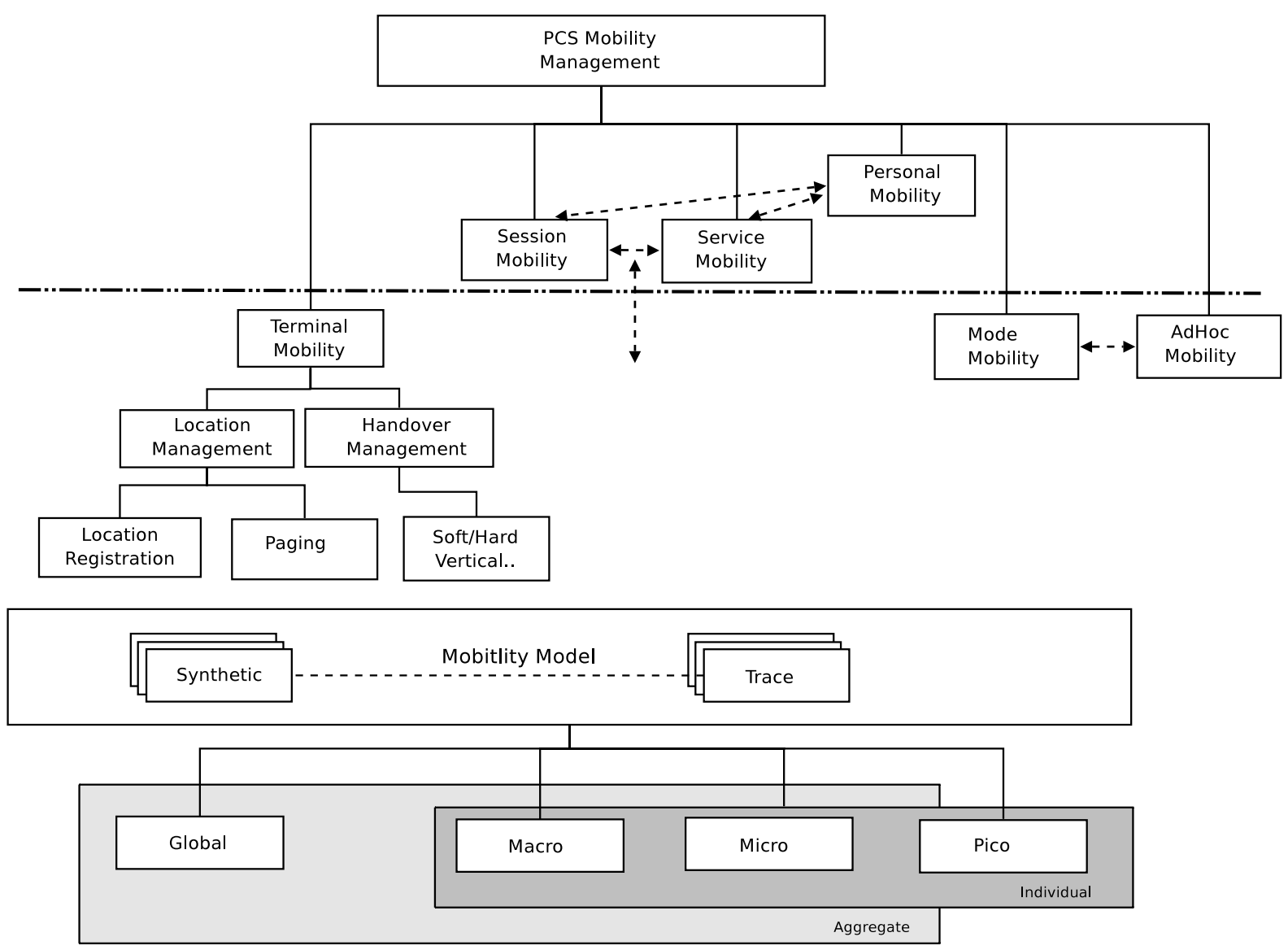

Fig. 7. Cellular Mobility Management is viewed as a two-tier solution, where the underlying mobility models can be provided by Synthetic or Trace models.

Finding the minimum of Eq. (23) (a detailed derivation and explanation of the parameter can be found in [?]) gives Eq. (24). Parameters in Eq. (26) is set according to Table 2 [?,?]. Setting the initial cost ratio $C_{t b f}$ to a non arbitrary value of 17 ensures the system has a cost ratio of registration to paging[?], taking into consideration the costs incurred by the fixed part of the network. The resulting minimum provides the optimum location area size of 29 for macro, 21 cells for micro and 45 cells for pico cell environment. The optimum location area size is calculated for macro, micro and pico cell environments and the results obtained are discussed in the following section.

$$
\begin{aligned}
C_{n t o t}(N)= & \rho N A_{\text {cell }} \lambda T_{p} B_{p}+ \\
& \frac{c \sqrt{N}}{N} \frac{\rho v_{x} L_{p r m}}{\pi} T_{u} B_{u}\left(1-E_{c}\right)
\end{aligned}
$$

Figure 8 graphically shows the obtained results for the different number of cells in the location area for macro, micro and pico sized cells. Figure 8(a) shows the result from a pico network scenario, Figs 8(b),(c) show the results from the micro and macro scenarios. The graphs show, in all scenarios, the paging cost increases steadily and directly proportional to the number of cells in a location area (as expected with a blanket paging scheme). The update cost curve is however of more interest, it follows an 
Table 2

Wireless Parameters for Location Area Optimisation algorithm

\begin{tabular}{lclll}
\hline Variable & Unit & Pico & Micro & Macro \\
\hline$v$ & $k m / h r$ & 1 & 5 & 20 \\
$E_{c}$ & Erlang & 0.04 & 0.04 & 0.04 \\
$\rho$ & User/ $/ m^{2}$ & 1 & 1 & 1 \\
$\lambda$ & Calls $/ \mathrm{hr}$ & 0.6 & 0.6 & 0.6 \\
$A_{\text {cell }}$ & $\mathrm{km}^{2}$ & 0.0265 & 0.65 & 10.39 \\
$C_{t b f}$ & - & 17 & 17 & 17 \\
\hline
\end{tabular}

asymptotical curve with high signalling cost in small location areas, but as $N$ increases the cost flattens out and approaches a cost limit. In comparison to the pico scenario, where both paging and signalling contributed to the overall cost without the either one being dominate, the macro and micro graphs shows the paging process dominating the total cost. The macro cell environment scenario had initially users travelling at a uniform velocity, the simulation was repeated with different values of velocity to study the influence on location area size. The velocity was incremented by $10 \mathrm{kmph}$ from an initial value of $10 \mathrm{kmph}$ to $120 \mathrm{kmph}$. As expected, increasing the velocity $v$ does not change the paging cost, but does impact the registration cost and therefore the overall signalling cost increases. Figure 8(d) shows the macro cell environment with two case scenarios: the velocity set to $10 \mathrm{kmph}$ and $120 \mathrm{kmph}$. It can be initially concluded that for fast moving nodes in a wireless cellular network, the number of cells in a location area must be increased to balance the registration and signalling cost.

The framework was used to compare with results to previous work [?] in obtaining an optimised area of 89 cells for a pico cell environment using the parameters outlined in [?]. Chih-Lin et al. obtained an optimised location area size of 93 cells. Reasons for the discrepancy in the results, and the cell count being high in comparison to this work, is that the authors omitted: (i) some users may be in an active state, (ii) standard 3GPP user/cell configurations, and (iii) fixed network cost.

\section{Conclusion}

Potential fluid dynamics provides a novel and flexible way of creating a user mobility model in wireless networks. The proposed model represents a situation where the users flow at certain speed and direction as a function of their current location. There are no random fluctuations in the user movement. The user density is assumed to be some constant $\rho$, and thus the mobility scenario gets solely defined by the velocity distribution of the fluid in the given area.

Consisting of five fundamentals building elements, the Free-stream, sink, source, doublet and vortex can be used in a linear combination to form any urban user trajectory in a pedestrian or lane section. Advantages of the model is the fact that it is simple to adapt to a given geographical topology (street section, pedestrians on a walkway etc.) and modelling desired mobility scenario by simply using the basic building blocks and taking advantage of the linearity of the solutions.

The PotFlow model distinguishes itself from other mobility models such as the Fluid model, or random walk models by generating smooth user paths or trajectories that have continuous varying velocity. The PotFlow model can recreate the Fluid mobility model by only having the Free-stream element present.

From a classification point of view the PotFlow model is an aggregate based synthetic mobility model. The model is considered to be an aggregate based model as it represents a collection of users and is not concerned with the movement of the individual. The model is regarded as a hybrid-synthetic model 


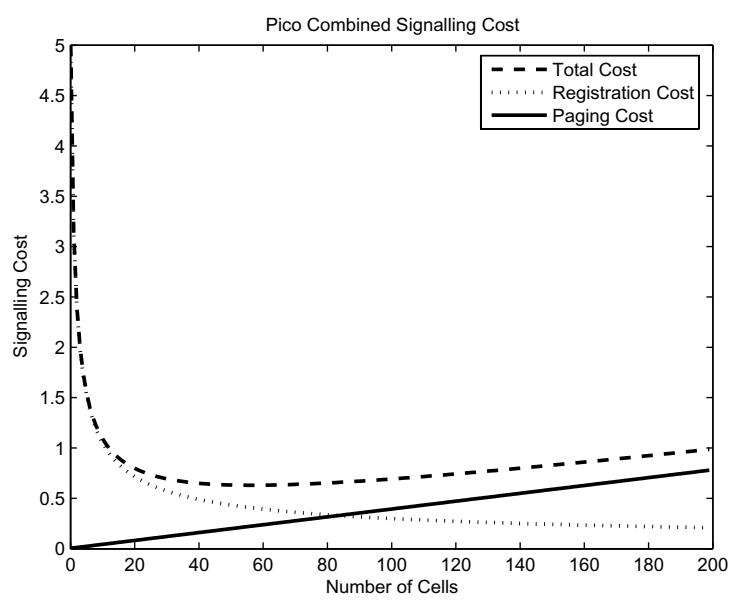

(a) Pico Cell

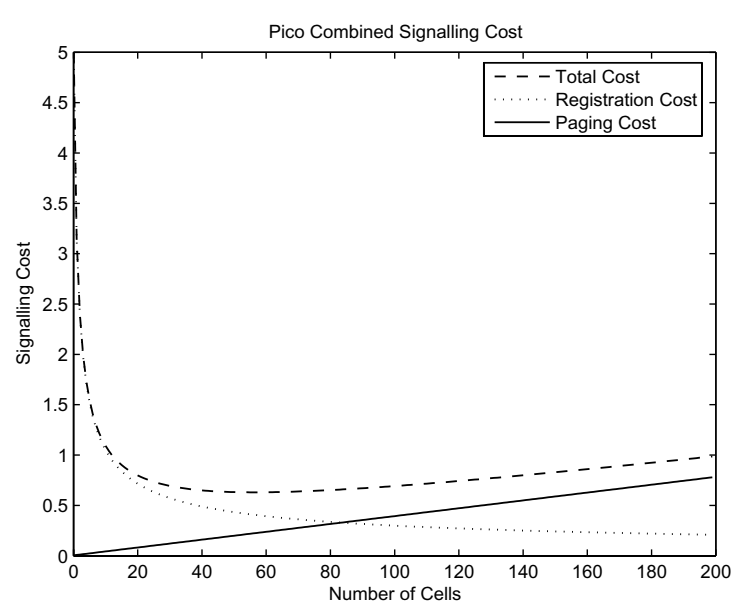

(b) Micro Cell

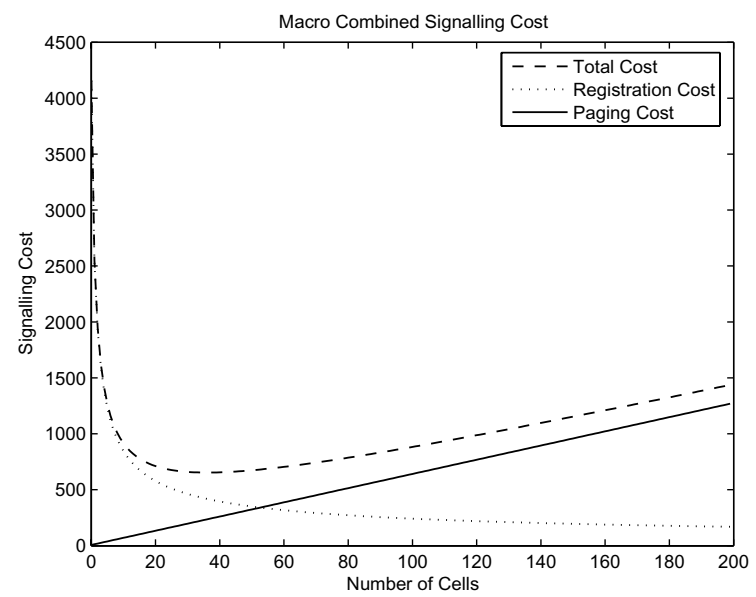

(c) Macro Cell

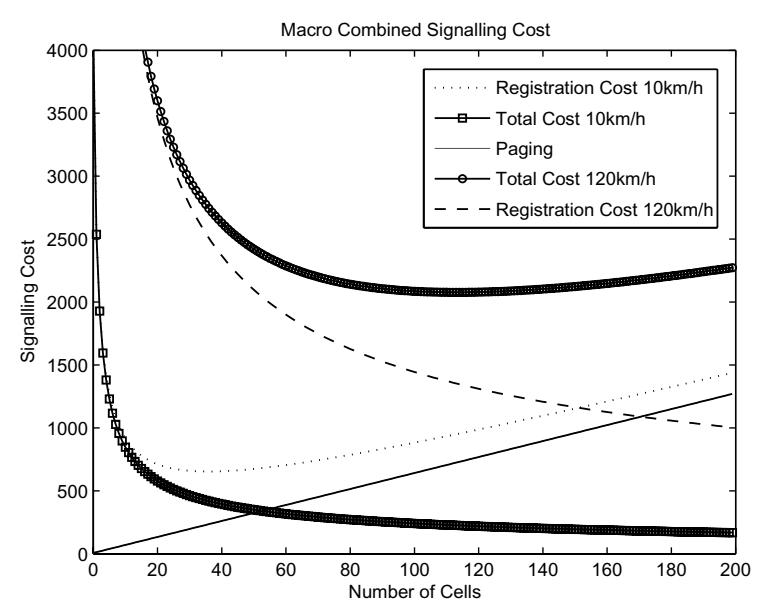

(d) Macro Limits

Fig. 8. Optimum Location Area (LA) for pico, micro, macro environments.

as its movement is bounded by environmental constraints such as walkways and lanes. The PotFlow model produces a two dimensional scenario plane and its smooth trajectory and varying velocity is more realistic to the way users behave on a macroscopic level than other previous mobility models. Multiple scenario planes can be layered on top of each other, which extends the model to provide user density levels of low, medium and high.

Applying the model to real urban scenarios is non-complex. Using an example of pedestrians on a walkway and commuters on a motorway, the density level, and movement characteristics of the commuter will differ for the pedestrian. To accommodate this PotFlow defines a motorway section and a pedestrian section also. Within the urban environments, all levels of mobility and density levels can exist concurrently and can be incorporated into the mobility model. The PotFlow mobility model accommodates these characteristics by tiling and layering solutions for pedestrian or motorway sections as illustrated by Fig. 9 . 

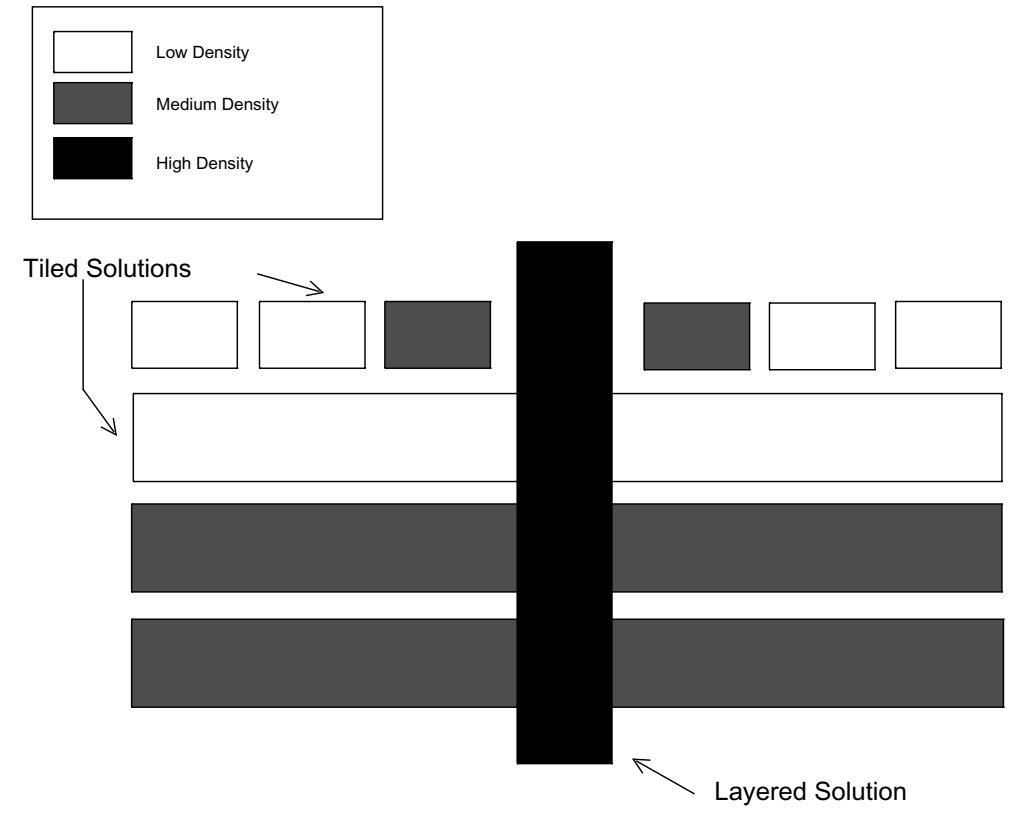

Fig. 9. The PotFlow model can replicate an urban environment by tiling and layer solutions.

Application of the mobility model is presented in this paper as a cellular network tool that can verify the dimensioning of user flows in an existing system. For example, in GSM systems, operators can measure from simulation the number of arriving and leaving inter-cellular handovers in each cell, they can also measure the number of location updates and thus estimate the street bounded user flow between cells (or location areas). Measurements of the pedestrian traffic in the cities are rare in the sense that in most cases only vehicular traffic is subject to measurements. The proposed mobility model is used in a wireless cellular network framework, with a terminal mobility management technique in place, in providing a method of calculating an optimised location area for pico, micro and macro cell environment.

\section{Acknowledgement}

This work was funded by the SFI NCNRC project (03/CE.2/I315).

\section{Appendix}

To derive the stream and velocity functions of the user, consider a closed surface $A$ whose position is fixed relative to the co-ordinate axes and which encloses a volume $V$ entirely occupied by fluid. If $\rho$ is the density of the fluid at position $x$ and at time $t$, the mass of the fluid enclosed by the surface at any instance is $\int \rho d V$ and the net rate at which mass is flowing outwards across the surface is $\int \rho \overrightarrow{v_{u}} \bullet \vec{n} d A$, where $\overrightarrow{v_{u}}$ is a velocity vector and $\delta V$ and $\delta A$ are the elements of the enclosed volume and of the area of the surrounding surface respectively. In the absence of sources of fluid the mass of the fluid is conserved so that:

$$
\frac{d}{d t} \int \rho d V=-\int \rho \overrightarrow{v_{u}} \bullet \vec{n} d A
$$


which, on differentiation under the integral sign and transformation of the surface integral may be written as:

$$
\int\left\{\frac{\partial \rho}{\partial t}+\nabla \bullet\left(\rho \overrightarrow{v_{u}}\right)\right\} d V=0
$$

This relation is valid for all choices of the volume $V$ lying entirely in the fluid, which is possible only if the integrand is identically zero everywhere in the fluid, hence:

$$
\frac{\partial \rho}{\partial t}+\nabla \bullet\left(\rho \overrightarrow{v_{u}}\right)=0
$$

where $\overrightarrow{v_{u}}$ is a velocity vector:

$$
\overrightarrow{v_{u}}=u(x, y, z, t) \hat{i}+v(x, y, z, t) \hat{j}+w(x, y, z, t) \hat{k}
$$

Where $x, y, z$ are in the space domain, and $t$ is in the time domain. Using Eq. (30) and expanding Eq. (29) gives the Continuity Equation:

$$
\frac{\partial \rho}{\partial t}+\frac{\partial(\rho u)}{\partial x}+\frac{\partial(\rho v)}{\partial y}+\frac{\partial(\rho w)}{\partial z}=0
$$

Hughes et al. [?] have investigated the flow of human crowds under the condition where $\rho$ is not constant and reflects the crowds discomfort level. Hughes et al. have incorporated $\rho$ to dictate the speed of the user in a crowd. In contrast in this paper, dealing with urban environments, compressing users is not considered which follows the third assumption. Using the fact $\rho$ is a constant simplifies Eq. (31):

$$
\frac{\partial u}{\partial x}+\frac{\partial v}{\partial y}+\frac{\partial w}{\partial z}=0
$$

Since this model only considers two dimensional movement Eq. (32) can be further simplified.

$$
\frac{\partial u}{\partial x}+\frac{\partial v}{\partial y}=0
$$

Revisiting the partial derivative of the Conservation of Mass rule, Eq. (33) can be expressed as:

$$
\nabla \bullet \vec{v}_{u}=0
$$

Herein, Eq. (34) shall be called the Continuity Rule, in order to be consistent with Fluid Mechanics. So for a two dimensional incompressible flow, the stream function $\psi$ can be defined to satisfy the Continuity Rule Eq. (34):

$$
\frac{\partial \psi}{\partial y}=u ;-\frac{\partial \psi}{\partial x}=v
$$

Defining $u$ and $v$ in this way automatically satisfies the Continuity Rule. It must be noted that $\psi$ only exists in two dimensional flows or axi-symmetric flows. Substituting the stream function into Eq. (33) results in:

$$
\frac{\partial^{2} \psi}{\partial x^{2}}+\frac{\partial^{2} \psi}{\partial y^{2}}=0
$$


Using the Laplacian operator this can be written as:

$$
\nabla^{2} \psi=0
$$

In this format Eq. (37) is known as the Laplace equation of the stream function. In a similar manner the Laplace equation for the velocity potential $\phi$ can be derived.

Consider a flow with zero vorticity $(\xi)$ i.e. $\xi=\nabla \times \vec{v}=0$, expanding this out gives:

$$
\xi=\nabla \times \vec{v}=\left|\begin{array}{ccc}
\hat{i} & \hat{j} & \hat{k} \\
\frac{\partial}{\partial x} & \frac{\partial}{\partial y} & \frac{\partial}{\partial z} \\
\frac{\partial \phi}{\partial x} & \frac{\partial \phi}{\partial y} & \frac{\partial \phi}{\partial z}
\end{array}\right|
$$

Further expansion of Eq. (38) gives:

$\xi=\nabla \times \vec{v}=$

$$
\hat{i}\left(\frac{\partial^{2} \phi}{\partial y \partial z}-\frac{\partial^{2} \phi}{\partial z \partial y}\right)+\hat{j}\left(\frac{\partial^{2} \phi}{\partial z \partial x}-\frac{\partial^{2} \phi}{\partial x \partial z}\right)+\hat{k}\left(\frac{\partial^{2} \phi}{\partial x \partial y}-\frac{\partial^{2} \phi}{\partial y \partial x}\right)
$$

Since the flow is irrotational and viscosity is not present the following is true:

$$
\vec{v}=\nabla \phi
$$

In order to satisfy the vorticity condition the velocity potential $\phi$ can be defined as:

$$
\frac{\partial \phi}{\partial x}=u ; \frac{\partial \phi}{\partial y}=v
$$

Substituting into Eq. (39) gives:

$$
\nabla^{2} \phi=0
$$

This is the Laplace equation for the velocity potential which is solved in Section 3.3 to provide the FreeStream, Vortex, Doublet, Source and Sink Blocks.

\section{References}

[1] D.C. Cox, Wireless personal communications - what is it? IEEE Personal Communications 2(2) (April 1995), 20-35.

[2] T. Camp, J. Boleng and V. Davies, A survey of mobility models for ad hoc network research, Wireless Communications and Mobile Computing (WCMC): Special issue on Mobile Ad Hoc Networking: Research, Trends and Applications 2(5) (2002), 483-502.

[3] C.K. Toh, Performance evaluation of crossover switch discovery algrothims for wireless ATM LANS, in: Proceedings of INFOCOM, 1996, pp. 1380-1387.

[4] C. Bettstetter, H. Hartenstein and X. Perez-Costa, Stochastic properties of the random waypoint mobility model: Epoch length, direction istribution, and cell change rate, in: Proceedings of the MSWiM'02 Atlanta, Georgia, USA, 2002.

[5] S. Phokeewanichkul, K. Chamnanya and B. Homnan, An analysis of mobility model in cellular communication system using gaussian probability density function for handover direction, in: Proceedings of the IASTED International Conference on Circuits, Signals, and Systems, May, 2004, pp. 378-383.

[6] B. Jabbari, Y. Zhou and F. Hillier, Random walk modeling of mobility in wireless networks, in: Proceedings of the 1998 48th IEEE Vehicular Technology Conference, VTC'98. Part 1 (of 3), 1998.

[7] J.W. Cohen, Analysis of Random Walks, (Vol. 2) of Studies in Probability, Optimization and Statistics, Ios Press Inc, 1992. 
[8] M.M. Zonoozi and P. Dassanayake, User mobility modeling and characterization of mobility patterns, IEEE J Select Areas Commun 15(7) (Sept. 1997), 1239-1252.

[9] R. Skehill and S. McGrath, Mobility modelling: A fluid dynamics approach, in: Proceedings of the 60th Vehicular Technology Conference, 2004.

[10] L.F. Henderson, On the fluid mechanics of human crowds, Transportation Research 8 (1974), 509-515.

[11] D. Marchal, Simulating pedestrian crowd behaviour in virtual cities, Technical report, Department of Computer Science, University College London, April 2002.

[12] R.L. Hughes, A continuum theory for the flow of pedestrians, Transportation Research Part B 36 (2002), 507535.

[13] S.P. Hoogendoorn and P.H.L. Bovy, Continuum modeling of multiclass traffic flow, Transportation Research Part B: Methodological 34(2) (2000), 123-146.

[14] S.P. Hoogendoorn and P.H.L. Bovy, Generic gas-kinetic traffic systems modeling with applications to vehicular traffic flow, ransportation Research Part B: Methodologica 35(4) (2001), 317-336.

[15] D. Helbing, Modeling multi-lane traffic flow with queuing effects, Physica A 242 (1997), 175-194.

[16] G.K. Still, Crowd Dynamics, Doctorate of philosophy, University of Warwick, Department of Mathematics, 2000.

[17] G.K. Batchelor, An Introduction to Fluid Mechanics, Cambridge University Press, 1967.

[18] Transportation Research Board, Highway capacity manual, National Research Council, Washington D.C., 2000.

[19] J.J. Fruin, Pedestrian Planning and Design, Metropolitan Association of Urban Designers and Environmental Planners, 1971.

[20] C. Abondo and S. Pierre, Dynamic location and forwarding pointers for mobility management, International Journal of Mobile Information Systems 1(1) (2005), 3-24.

[21] B. Kovacs, M. Szalay and S. Imre, Modelling and quantitative analysis of ltrack - a novel mobility management algorithm, International Journal of Mobile Information Systems 2(1) (2006), 3-24.

[22] V.H. MacDonald, The cellular concept, Bell System Tech. Journal 58(1) (January 1979), 15-41.

[23] V. Wong and V. Leung, Location management for next generation personal communication networks, IEEE Network 14(5) (September 2000), 8-14.

[24] R.J. Skehill and S. McGrath, PCS location area optimisation using an aggregate mobility model, in: Proceedings of the IEEE Personal,Indoor And Mobile Radio Communications, Barcelona, September 2004.

[25] G.P. Pollini and S. Tabbane, Intelligent network signaling and switching cost of an alternative location strategy using memory, in: Proceedings of the IEEE Vechicular Technology Conference, VTC'93, May 1993, pp. 931-934.

[26] ETSI, Tr 101112 v3.2.0 selection procedures for the choice of radio transmission technologies of the UMTS: UMTS $30.03,1998$.

[27] I. Chih-Lin, G.P. Pollini and R.D. Gitlin, Pcs mobility management using the reverse virtual call setup algorithm, IEEE/ACM Transactions on Networking (TON) 5(1) (1997), 13-24.

[28] R.L. Hughes, The flow of human crowds, Annu Rev Fluid Mech 35 (2003), 169-182. 

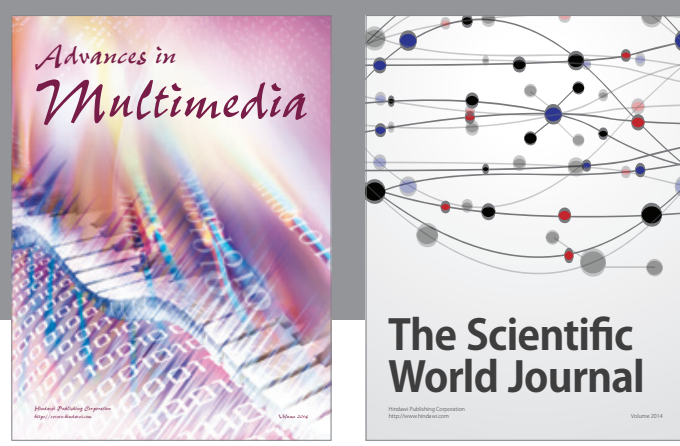

The Scientific World Journal
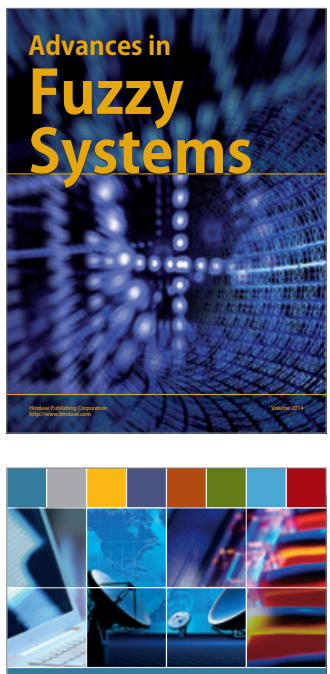

Computer Networks and Communications
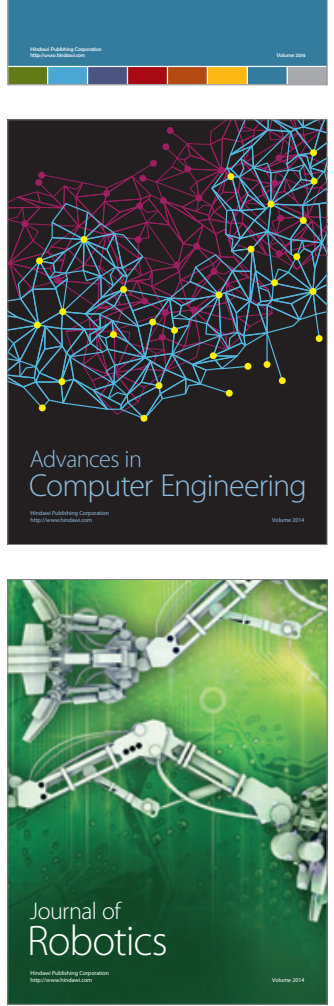
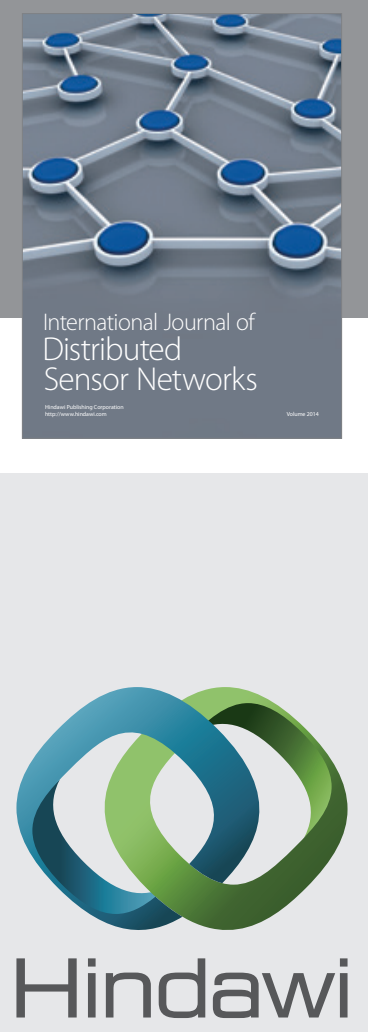

Submit your manuscripts at

http://www.hindawi.com
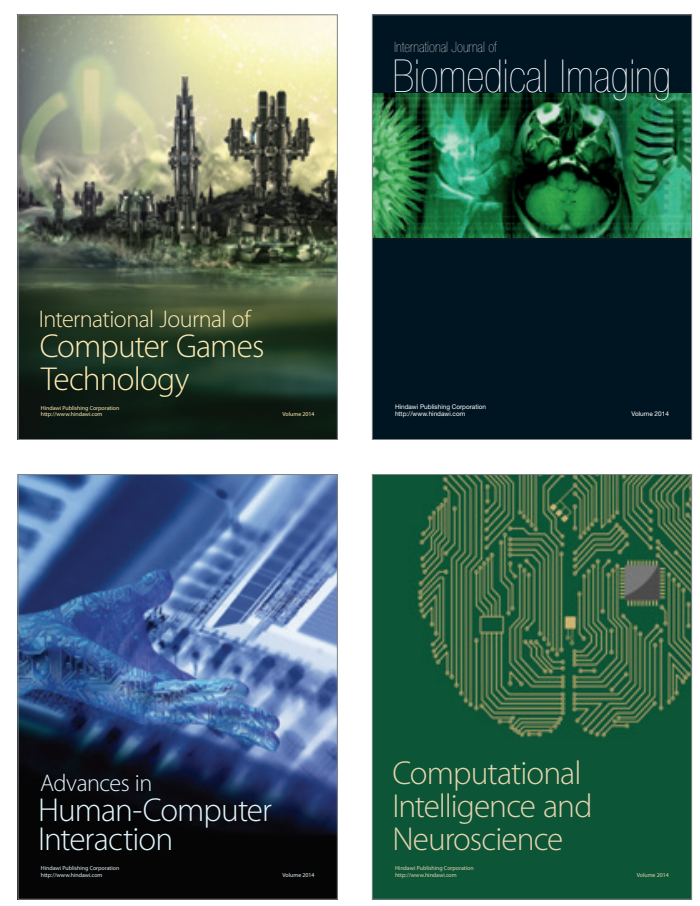
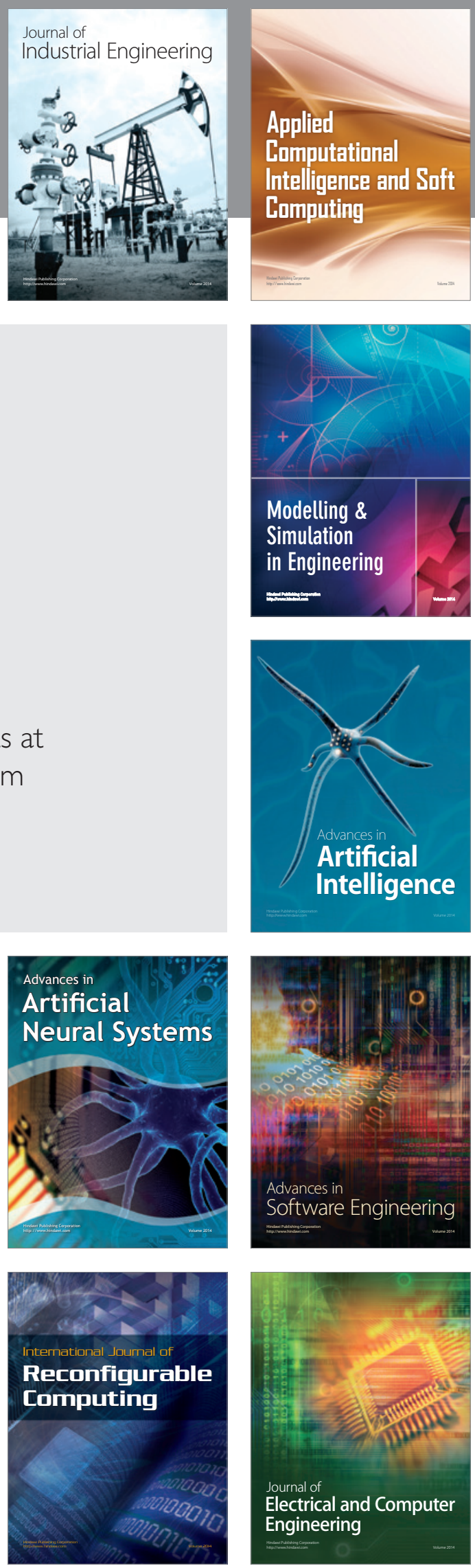